\title{
Pflaster statt Messer - neue Therapie des Basalioms mit 5-Fluorouracil?
}

\author{
Christiane Bayerl \\ D. Siebold \\ M. Fuchslocher \\ E. G. Jung
}

\section{Plaster Instead of Knife - New Therapy of Basal Cell Carcinoma with \\ 5-Fluorouracil?}

\section{Zusammenfassung}

Das Basaliom ist der häufigste epitheliale Hauttumor des Menschen und wird zumeist chirurgisch behandelt. Wir stellen drei Kasuistiken zur lokalen Behandlung histologisch gesicherter Basaliome mit 5-Fluorouracil in einem transdermalen System vor, das täglich erneuert wird. Die Behandlung war sicher und effektiv, wie die Biopsien und die Kontrollen bis 6 Monate nach Therapieende zeigten. Eine Irritation der Haut trat nicht auf. Die kosmetischen Ergebnisse waren hervorragend. Daher haben wir eine klinische Studie geplant, um die Effekte des 5-FluorouracilPflasters als neue Therapieoption für das Basaliom zu überprüfen.

\section{Abstract}

Basal cell carcinoma is the most frequent non-melanoma skin cancer in humans, treated mostly surgically. We present three case reports about topical application of 5-fluorouracil in a transdermal delivery system applied daily on histologically proven basal cell carcinomas. The treatment was safe and effective, shown by biopsies and follow-up for 6 months. Irritative effects were not seen. The cosmetic results were excellent. According to this, we will perform a controlled study to evaluate if 5-fluorouracil plaster might be a new treatment option for basal cell carcinoma.

Basaliomen ist limitiert durch grippeähnliche Nebenwirkungen, Anämie, Neutropenie oder Thrombozytopenie [12,24].

Ziel dieser drei Kasuisitken war es, das Ansprechen des Basalioms auf eine Zubereitung des 5-Fluorouracils in einem transdermalen System zu beobachten mit der Perspektive, ggf. in der Folge eine klinisch kontrollierte Studie aufzulegen. 5-Fluorouracil (5-FU) ist ein Pyrimidinderivat mit zytostatischer Wirkung. Als Antimetabolit des Uracils blockiert es die intrazelluläre Konversion von Nukleotiden bei der DNA-Synthese. So wird der Abschluss des Zellzyklus mit der DNA-Synthesephase der Zelle angehalten und dadurch die Zellproliferation verhindert. Als antineoplastischer Wirkstoff zur Therapie von Tumoren ist die Substanz seit langem bekannt für gastrointestinale, Mamma- und Blasen-Tumoren und Tumoren des Hals- und Nackenraumes $[3,9]$. Zugelassen ist eine Creme zur Therapie der aktinischen Keratose und des Morbus Bowen [10] und eine Lösung zur Warzenbehandlung [2]. Diese lokalen 5-FU-Zubereitungen führen zu ei-

Institutsangaben

Klinik für Dermatologie, Venerologie und Allergologie Mannheim, Universitätsklinik Mannheim

Korrespondenzadresse

PD Dr. med. Christiane Bayerl · Klinik für Dermatologie, Venerologie und Allergologie Mannheim .

Universitätsklinik · Theodor-Kutzer-Ufer 1 - 3 · 68135 Mannheim •

E-mail: christiane.bayerl@haut.ma.uni-heidelberg.de 


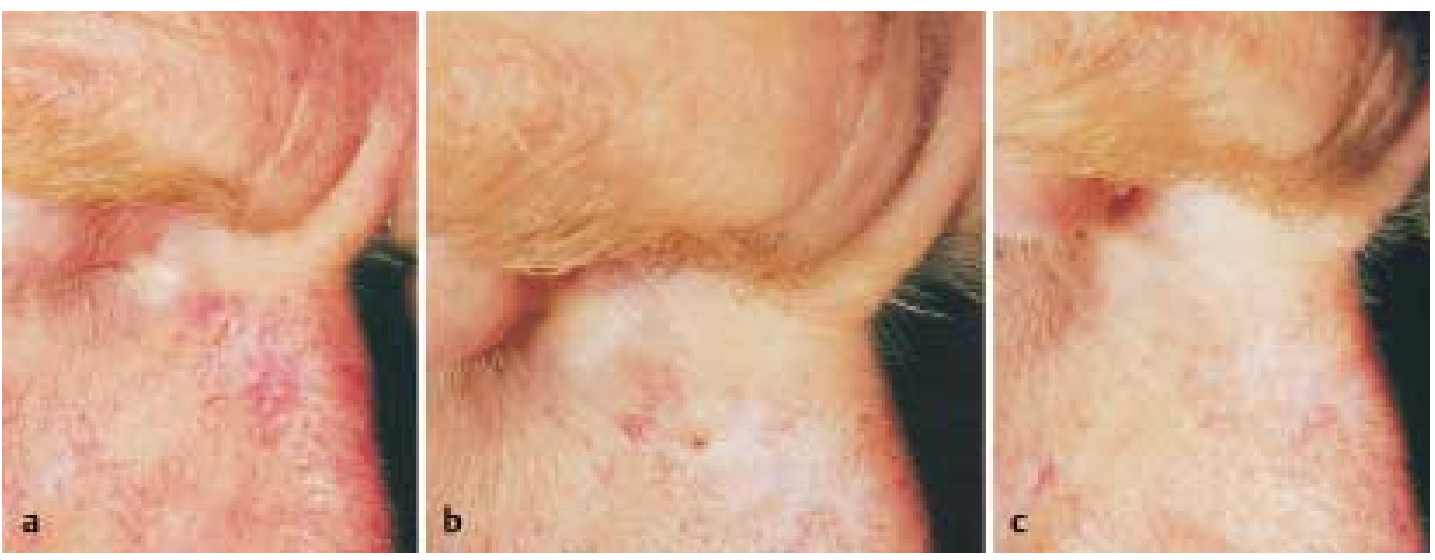

Abb. 1 Basaliom im Augenwinkel a vor Therapiebeginn, $\mathbf{b}$ nach 3 Wochen der Behandlung mit 5-Fluorouracil-Pflaster, $\mathbf{c} 6$ Monate nach Abschluss der Behandlung, weiterhin abgeheilt, kein Rezidiv.
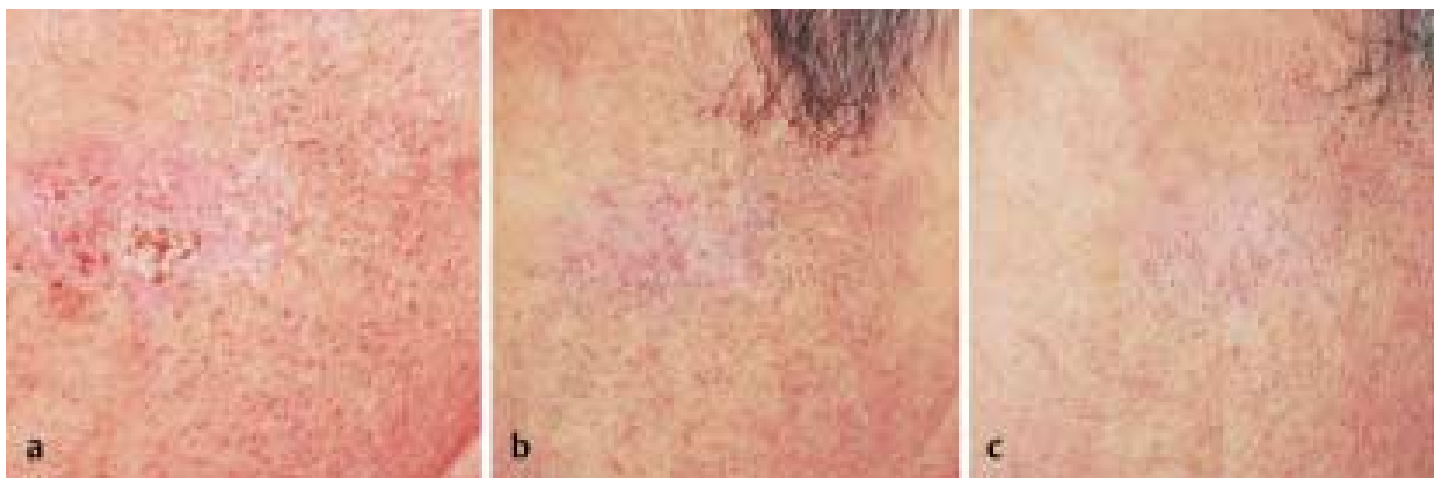

Abb. 2 Basaliom an der linken Wange im Bartbereich a vor Therapiebeginn, b Kontrolluntersuchung 1 Monat nach Therapieende und c 6 Monate nach der Behandlung kosmetisch sehr gutes Ergebnis.

nem Therapieerfolg, jedoch über starke entzündliche Reaktionen. Es sollte daher eine Therapieform gefunden werden, die weniger irritative Hautreizung und Entzündung auslöst und dennoch effektiv ist. Hierzu wurde ein 5-FU-Pflaster, das bisher die Zulassung für aktinische Keratosen hatte und einen niedrigeren Wirkstoffgehalt $\left(85 \mu \mathrm{g} / \mathrm{cm}^{2}\right.$ 5-Fluorouracil) als die marktüblichen Cremes aufweist, eingesetzt.

\section{Kasuistiken}

Die drei 3 Kasuistiken beschreiben individuelle Heilversuche. Beobachtet wurde der Heilungsverlauf bei täglicher Applikation eines 5-FU-Pflasters (5-FU-TOP, Fa. LTS Lohmann Therapie-Systeme AG, Andernach) über 3 Therapiewochen und 1, 3, 6 Monate nach Therapieende mit histologischer Untersuchung. Ein Basalzell-Naevus-Syndrom, Xeroderma pigmentosum, Röntgenschaden, Arsen oder Teerexposition lagen nicht vor. Es handelte sich um primäre, histologisch gesicherte, nicht vorbehandelte Basaliome. Die Patienten hatten den Wunsch nach einem konservativen Therapieverfahren. Das Pflaster wurde in täglichem Wechsel über die drei Behandlungswochen aufgetragen. Der Heilungsverlauf wurde in dieser Zeit wöchentlich vom behandelnden Arzt der Ambulanz der Klinik für Dermatologie kontrolliert. Zusätzlich wurde die lokale Verträglichkeit beobachtet.

\section{Kasuistik 1}

69-jähriger Patient mit Zufallsbefund eines histologisch als sklerodermiform befundeten Basalioms im rechten Nasen/Augenwinkel bei Vorstellung in der Sprechstunde aus anderen Gründen. Bei Studienbeginn (Abb.1a), nach 3 Therapiewochen
(Abb.1b) und 6 Monate nach Therapieabschluss (Abb.1c). Zu diesem Zeitpunkt histologisch kein Basaliom nachweisbar. Kontrolle weiter angeraten.

\section{Kasuistik 2}

65-jähriger Patient mit Vorstellung wegen einer beim Rasieren seit 2 Monaten immer wieder blutenden Stelle an der linken Wange. Histologisch als solides Basaliom gesichert. Bei Studienbeginn (Abb. 2a), 1 Monat nach Therapieende Patient mit neu gewachsenen Haar-Koteletten (Abb. 2 b) und 6 Monate nach Therapieende (Abb. 2c). Stanzbiopsie mit massiver aktinischer dermaler Elastose sowie mäßiger epidermaler Atrophie.

\section{Kasusitik 3}

77-jähriger Patient mit solidem, nodulären histologisch gesicherten Basaliom unterhalb der rechten Klavicula. Bei Studienbeginn (Abb.3a), nach 3 Therapiewochen (Abb.3b), 1 Monat nach Therapieende (Abb.3c), 3 Monate nach Therapieende (Abb.3d), 6 Monate nach Therapieende (Abb.3e). Eine Stanzbiopsie 1 Monat nach Therapieende zeigte eine aktinische Elastose sowie Fibrose der von schütteren rundzelligen Entzündungsinfiltraten sowie vereinzelten Melanophagen durchsetzten Dermis. Kein Nachweis eines Basalioms.

\section{Diskussion}

Eine erfolgreiche topische Therapie von Basaliomen ist für den Patienten angenehm, risikoarm und erspart eine Operation. Die Patienten unserer drei Kasuistiken profitierten von diesen Vorteilen einer lokalen 5-FU-Therapie in Form eines Transdermalen 


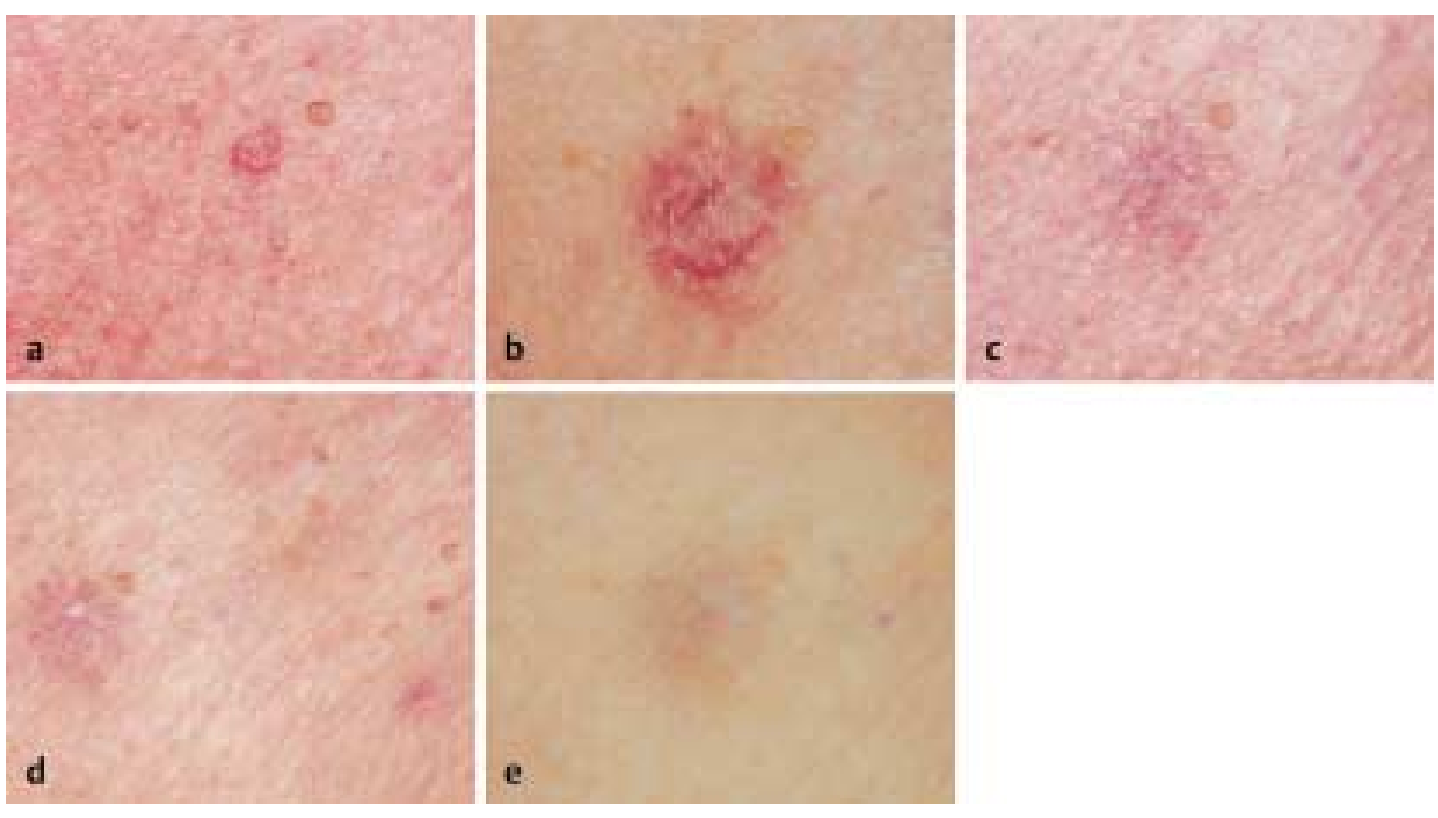

Abb. 3 Basaliom intraklaviculär a bei Therapiebeginn, b nach 3 Wochen der Behandlung Abschluss der Therapie, c 1 Monat nach Therapieende vor Entnahme der Biopsie zur Kontrolle des Therapieerfolges: kein Basaliom mehr. d 3 Monate nach Therapieabschluss, e 6 Monate nach Therapieabschluss, kein Rezidiv.

Systems (TDS). Eine Entzündung trat nicht auf unter der 5-FUPflastertherapie. Das kosmetische Ergebnis war ausgesprochen zufriedenstellend.

Studien zu verschiedenen Zubereitungen und Therapieschemata zu 5-Fluorouracil liegen bereits vor. In einer Pilotstudie verbesserte die Zugabe von Phosphatidylcholin als Carrier zu topisch appliziertem 5-Fluorouracil die Heilungsrate von 57\% auf 90\% [22]. Eine Zubereitung von 5-Fluorouracil in Form eines viskösen injizierbaren Gels mit Epinephrin zeigte bei 3-mal wöchentlicher Anwendung über 14 Tage ein 100\%iges Ansprechen [15] mit dem Nachteil für den Patienten, dass Injektionen notwendig waren. Topisches Tretinoin zur Behandlung von Basaliomen war dagegen bei weitem nicht so effektiv wie 5-FU [19]. Weitere Studien zur topischen Therapie des Basalioms liegen vor zu Imiquimod, einer Substanz, die vor allem die zelluräre Immunität beeinflusst. In einer multizentrischen Studie als 5\%ige Creme war Imiquimod effektiv, zeigte aber dosisabhängig ausgeprägte Hautreizungen [13].

Möglicherweise bedingt gerade die Okklusion, dass 5-FU als TDS trotz geringer Wirksubstanz gute Effizienz bei unseren drei Patienten gezeigt hatte. Okklusive Verbände bei Behandlung mit 5-FU hatten sich bereits in den 60er Jahren bewährt [25]. Das hier verwendete 5-FU-Pflastersystem hat einen Wirkstoffgehalt von $85 \mu \mathrm{g} / \mathrm{cm}^{2}$. Die auf die Haut einwirkende Sustanzmenge liegt bei ca. $25-50 \mu \mathrm{g} / \mathrm{cm}^{2} / 24 \mathrm{~h}$. Bei der Anwendung einer $5 \%$ igen 5-FU Salbenzubereitung wird als tägliche lokale Dosis 25-50 mg 5-FU appliziert. Der therapeutische Effekt lässt sich also nur über die verbesserte Aufnahme durch die Haut über das Pflaster als TDS verstehen. TDS erhöhen die Permeation einer Substanz durch die Haut, indem sie die Oberflächenbarriere der Epidermis verändern $[14,17]$. Ein Risiko der TDS ist das Strippen des Stratum corneums durch den täglichen Pflasterwechsel oder Unverträglichkeiten und Allergien auf Klebstoffe oder Pflasterinhaltsstoffe. Unsere Patienten hatten das TDS gut vertragen. Zudem stehen zur Zeit objektive Evaluierungssysteme bereit, um sowohl die Haftfähigkeit eines solchen Pflasters als auch die Verträglichkeit zu überprüfen $[1,5]$.
Die Lokaltherapie der Basaliome ist für ausgewählte Patienten eine gute Alternative. Sie erspart Angst vor einer Operation, Schmerzen bei der Operation und während der Wundheilung. Einigen Patienten mit psychischen Störungen, mit orthopädischen Beschwerden und verschiedenen altersbedingten Erkrankungen ist es nicht möglich, auf einem Operationstisch für längere Zeit zu liegen. Auch für Patienten mit Marcumartherapie oder Unverträglichkeitsreaktionen auf Lokalanästhetika ist diese Therapieform vorzuziehen. Hinzu kommt der kosmetische Aspekt mit einem ausgesprochen ansprechenden Ergebnis nach Therapieabschluss.

Über Effektivität, Langzeitverlauf und Rezidivraten lässt sich aufgrund der beschriebenen Kasuistiken keine Aussage machen. Bisher gezeigt wurde, dass eine 5-FU/Salicylsäurelösung bei zwei Drittel von 24 behandelten Patienten zu keiner Heilung geführt hatte [6]. Die 5-Jahres-Rezidivraten lagen bei erstmalig behandelten Basaliomen nach Mohs' mikrographischer Chirurgie bei $1 \%$, nach chirurgischer Exzision bei $5 \%$ - 10\%, nach Kürettage und Elektrodessication bei $7 \%-13 \%$, nach Kryochirurgie bei $3 \%-7 \%$, nach Radiatio bei $7 \%-8 \%$ und nach Immunotherapie mit 5-FU-Creme bei $21 \%[20,23,26]$. Daran muss sich jede neue Therapieform messen. Dies sind Gründe, die uns bewegt haben, eine prospektive, zur Zeit bereits laufende Studie zur Untersuchung der Effektivität und Verträglichkeit dieser Therapieform durchzuführen.

\section{Literatur}

${ }^{1}$ Bodde HE, Verhoeven J, van Driel LM. The skin compliance of transdermal drug delivery systems. Crit Rev Ther Carrier Syst 1989; 6: 87-115

${ }^{2}$ Bunney MH, Nolan MW, Williams DA. An assessment of methods of treating viral warts by comparison of treatment trials based on a standard design. Br J Dermatol 1976; 94: 667-679

${ }^{3}$ Cartei F, Carteil G, Girali T et al. A phase II study of oral fluorouracil for gastrointestinal cancer. Anticancer Drugs 1996; 7: 48-53

${ }^{4}$ von Domarus H, Stevens PJ. Metastatic basal cell carcinoma. Report of five cases and review of 170 cases in the literature. J Am Acad Dermatol 1984; 10: $1043-1060$ 
${ }^{5}$ Ehrlich A, Henkerl-Ernst J, Schaefer A, Assmussen B, Lucker PW. Therapeutic delivery systems: a new approach to evaluate physical properties of transdermal delivery systems (TDS). Methods Find Exp Clin Pharmacol 1999; 21: 69-71

${ }^{6}$ Engelberg IS, Ronnen M, Suster S, Schewach-Millet M, Stempler D, Schibi-Brilliant G. Effects of Solcoderm. Int J Dermatol 1986; 25: 606-607

${ }^{7}$ Glorer E, Rzany B, Jung EG. Ausgedehnt exulzeriertes Basaliom. Behandlung mit ionisierender Strahlung nach elektrokaustischer Abtragung. Hautarzt 2001; 52: 646-648

8 Graham G. Statistical data on malignant tumors in cryosurgery. J Dermatol Surg Oncol 1983; 9: 238-239

${ }^{9}$ Grunberg SM, Clay C, Spicer DV. Tolerance of extended (28 day) continuous infusion of 5-fluorouracil in advanced head and neck cancer. Scl Cancer Ther 1991; 7: 17-21

${ }^{10}$ Gupta AK, Weiss JS, Jorizzi JL. 5-fluorouracil 0,5\% cream for multiple actinic keratoses of the face and anterior scalp. Skin Therapy Lett 2001; 6: $1-4$

${ }^{11}$ Holt PJA. Cyotherapy for skin cancer: results over a 5 year period using liquid nitrogen spray cryosurgery. Br J Dermatol 1988; 119: 231-240

${ }^{12}$ Kowalzick L, Rogozinski T, Schober $\mathrm{C}$ et al. Treatment of basal cell carcinoma with intralesional recombinant interferon beta: a dose-finding study. Eur J Dermatol 1994; 4: 430-433

${ }^{13}$ Marks R, Gebauer K, Shumack S, Amies M, Bryden J, Fox TL, Owens ML. Imiquimod $5 \%$ cream in the treatment of superficial basal cell carcinoma: results of a multicenter 6-week dose-response trial. J Am Acad Dermatol 2001; 44: 807-813

${ }^{14}$ Merkle HP. Transdermal delivery systems. Methods Find Exp Clin Pharmacol 1989; 11: 135-153

${ }^{15}$ Miller B, Shavin J, Cognetta A et al. Nonsurgical treatment of basal cell carcinomas with intralesional 5-fluorouracil/epinephrine injectable gel. J Am Acad Dermatol 1997; 36: 72 - 77

${ }^{16}$ Mohs FE. Chemosurgery for the microscopically controlled excision of skin cancer. J Surg Oncol 1971; 3: 257-267

17 Niedner R. Transdermal therapeutic systems (TTS). Hautarzt 1988; 39: 761 - 766

18 Orton CI. The treatment of basal cell carcinoma by radiotherapy. Clin Oncol 1978; 4: 317-322

${ }^{19}$ Peck GL. Topical tretinoin in actinic keratosis and basal cell carcinoma. J Am Acad Dermatol 1986; 15: 829-835

${ }^{20}$ Reyman F. Treatment of basal cell carcinoma of the skin with 5-fluorouracil ointment: a 10-year follow-up study. Dermatologica 1979; 158: $368-372$

${ }^{21}$ Reyman F. 15 years' experience with treatment of basal cell carcinomas of the skin with curettage. Acta Derm Venereol 1985; 120: 56 - 59

${ }^{22}$ Romagosa R, Saap L, Givens M, Salvarrey A, He JL, Hsai SL, Taylor JR. A pilot study to evaluate the treatment of basal cell carcinoma with 5-fluorouracil using phosphatidyl choline as a transepidermal carrier. Dermatol Surg 2000; 26: $338-340$

${ }^{23}$ Rowe DE, Caroll RJ, Day CL Jr. Long-term recurrence rates in previously untreated (primary) basal cell carcinoma: implications for patient follow-up. J Dermatol Surg Oncol 1989; 15: 315-328

24 Stenquist B, Wennberg AM, Gisslen H, Larko O. Treatment of aggressive basal cell carcinoma with intralesional interferon: evaluation of efficacy by Mohs surgery. J Am Acad Dermatol 1992; 27: 65-69

${ }^{25}$ Stoll HL Jr, Klein E. Tumors of the skin. XI. Effect of occlusive dressing on the local administration of 5-fluorouracil to superficial basal cell carcinoma. J Invest Dermatol 1969; 52: $304-306$

${ }^{26}$ Thissen M, Neumann M, Schouten L. A systematic review of treatment modalities for primary basal cell carcinomas. Arch Dermatol 1999; 135: $1177-1183$

${ }^{27}$ Wang I, Bendsoe N, Klintenberg CAF, Enejder AM, Andersson-Engels S, Svanberg S, Svanberg K. Photodynamic therapy versus cryosurgery of basal cell carcinoma: results of a phase III clinical trial. Br J Dermatol 2001; $144: 832-840$

${ }^{28}$ Whelan C, Deckers P. Electrocoagulation for skin cancer: an old oncologic tool revisted. Cancer 1981; 47: 2280-2287

${ }^{29}$ Wolf P, Rieger E, Kerl H. Topical photodynamic therapy with endogenous porphyrins after application of 5-aminolevulinic acid. An alternative treatment modality for solar keratose, superficial squamous cell carcinomas and basal cell carcinomas? J Am Acad Dermatol 1993; 28: $17-21$

\section{Buchbesprechung}

\section{Laser und IPL-Technologie in der Dermatologie und Ästhetischen Medizin}

C. Raulin, Bärbel Greve

180 Seiten, 289 farbige Abb. Schattauer, Stuttgart 2001.

Geb. 101,-. ISBN 3-7945-2042-4

Nach einer leicht verständlichen Einführung in die Grundlagen der Technik der Laser und der ähnlich wirkenden Intensiv-PulsLichtquellen (IPL) werden in den folgenden 10 Kapiteln die dermatologischen und ästhetischen Indikationen für diese Geräte dargestellt. Unterstützt durch exzellentes, vom Umfang und der Qualität her schon einem Atlas würdiges Bildmaterial, sowie durch zahlreiche Tabellen werden die Möglichkeiten und Grenzen der Behandlung einer Vielzahl von Hautveränderungen abgehandelt. Die Fülle des Materials ist beeindruckend: selbst bei genauer Durchsicht fehlen auch seltene Indikationen nicht, ein Hinweis auf die gründliche Sichtung der einschlägigen Literatur. Im dritten Teil des Buches werden besondere Aspekte der Lasertherapie dargestellt. Besonders wichtig und verdienstvoll sind 2 Kapitel zu Behandlungsfehlern und zu ethischen Fragen der Lasertherapie, obwohl schon bei den einzelnen Entitäten auf Fehler und unerwünschte Wirkungen dieser Therapie hingewiesen wird. Die Fülle der dargestellten, differenzialdiagnostisch teils anspruchsvollen Hautveränderungen, die mit den verschiedenen Lasern und IPL-Geräten behandelt werden kann, macht unmissverständlich deutlich, dass die Lasertherapie der meisten Hautveränderungen besonders geschulten Hautärzten überlassen bleiben muss. Fazit: Aufgrund der ausführlichen und differenzierten Darstellung der Lasertherapie von Hautveränderungen und der exzellenten Bebilderung ist das Buch ein Muss für jeden lasertherapeutisch Engagierten, für Anfänger wie für Fortgeschrittene. Für letztere ist es zudem ein zuverlässiges Referenzwerk für Begutachtungen.

F. A. Bahmer, Bremen 